\title{
Bilateral adrenal incidentalomas and NR3C1 mutations causing glucocorticoid resistance: is there an association?
}

\author{
Nicolas C Nicolaides ${ }^{1,2}$ and George P Chrousos ${ }^{1,2}$ \\ ${ }^{1}$ Division of Endocrinology, Metabolism and Diabetes, First Department of Pediatrics, National and Kapodistrian \\ University of Athens Medical School, 'Aghia Sophia' Children's Hospital, Athens, Greece and 'Division of \\ Endocrinology and Metabolism, Biomedical Research Foundation of the Academy of Athens, Athens, Greece
}

Correspondence should be addressed to N C Nicolaides

Email

nnicolaides@bioacademy.gr

\begin{abstract}
Glucocorticoids signal through their cognate, ubiquitously expressed glucocorticoid receptor (GR), which influences the transcription of a large number of target genes. Several genetic defects, including point mutations, deletions or insertions in the NR3C1 gene that encodes the GR, have been associated with familial or sporadic generalized glucocorticoid resistance or Chrousos syndrome. One of the clinical manifestations of this rare endocrine condition is bilateral adrenal hyperplasia due to compensatory elevations of plasma ACTH concentrations. In this commentary, we discuss the interesting findings of the recently published French MUTA-GR study and present our perspective on the evolving field of NR3C1 pathology.
\end{abstract}

\section{Glucocorticoids in homeostasis and cacostasis}

Secreted by the adrenal cortices, glucocorticoids are major effector molecules of the stress response (1). These steroid hormones contribute substantially to the maintenance of our resting and stress-related homeostasis by influencing almost all physiologic functions, including arousal and sleep, memory, maintenance of cardiovascular tone, intermediary metabolism, the immune response and many others (2). The myriad of glucocorticoid effects are mediated by a ubiquitously expressed intracellular protein, the human glucocorticoid receptor (hGR), which influences the transcription rate of approximately one in five genes of the human leukocyte expressed genome (3, 4). In addition, the crosstalk of hGR with several other fundamental transcription factors, such as the nuclear


signal transducers and activators of transcription (STATs), allows indirect regulation of gene expression via their intracellular signaling systems (1, 2, 3). Furthermore, a growing body of evidence suggests that glucocorticoids

https://eje.bioscientifica.com https://doi.org/10.1530/EJE-18-0471
(C) 2018 European Society of Endocrinology Printed in Great Britain acting through their cognate receptor, may alter the methylation status of cytosine-guanine dinucleotides $(\mathrm{CpG})$ located in the regulatory regions of several genes, leading to important epigenetic alterations (5).

The hGR is encoded by the NR3C1 gene, which is located in the short arm of chromosome 5. This gene is composed of ten exons. Exon 1 contains several promoters of the gene, whereas exons $2-9 \alpha$ or $-9 \beta$ generate by alternative transcription of the two main receptor isoforms, the hGR $\alpha$ and the hGR $\beta$, respectively (6). Mutations, deletions or insertions in the NR3C1 gene have been associated with familial or sporadic primary generalized glucocorticoid resistance or Chrousos syndrome $(7,8)$. This is a rare condition, characterized by decreased sensitivity to glucocorticoids of all their target tissues, including those responsible for the glucocorticoid negative feedback loops, and leading to compensatory elevation of ACTH and cortisol secretion (9). To date, 26 different genetic defects have been identified in the 
NR3C1 gene $(10,11,12)$. The compensatory increase in plasma ACTH concentrations has been considered responsible for adrenal hyperplasia and hypersecretion of cortisol, steroid precursors with mineralocorticoid activity (e.g. deoxy-corticosterone and corticosterone) and adrenal androgens, such as androstenedione, DHEA and DHEAS (Fig. 1). Patients with primary generalized glucocorticoid resistance (PGGR) may be asymptomatic with only biochemical alterations or may present with clinical manifestations of mineralocorticoid excess (hypertension and/or hypokalemic alkalosis) and/or adrenal androgen excess, such as ambiguous genitalia, precocious puberty, cystic acne, hirsutism and hypofertility in both sexes (Fig. 1) (10, 11).

We and others have identified novel NR3C1 genetic defects in patients with a broad clinical spectrum of PGGR (reviewed in (11)). The progress of molecular and cellular biology has allowed detailed investigation of the molecular mechanisms through which defective receptors cause glucocorticoid resistance both in in vitro assays and in silico. We demonstrated that all defective GRs impaired many steps of glucocorticoid signal transduction depending on the position of a missense mutation, insertion or deletion in the NR3C1 gene (11). Interestingly, a number of patients with clinical and/or biochemical findings suggestive of PGGR do not harbor a mutant GR, indicating that several other genes play important roles in defining tissue sensitivity to glucocorticoids (11).
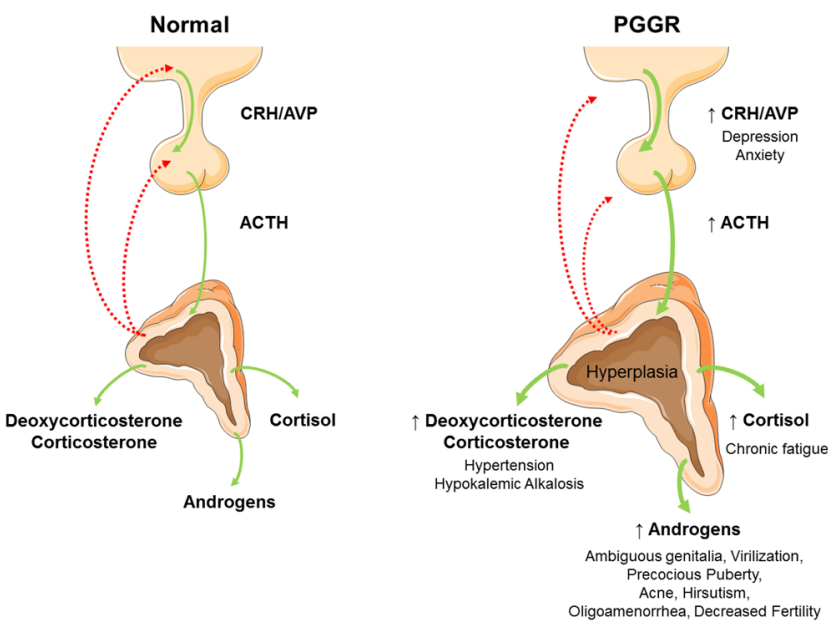

Figure 1

Pathophysiology and clinical manifestations of PGGR syndrome. ACTH, adrenocorticotropic hormone; AVP, argininevasopressin; $\mathrm{CRH}$, corticotropin-releasing hormone; PGGR, primary generalized glucocorticoid resistance. Modified from Reference (10) with permission.

\section{Lessons from the French MUTA-GR study}

A recently published elegant French study provided interesting results on the association between bilateral adrenal hyperplasia and NR3C1 mutations (12). Vitellius et al. estimated the prevalence of NR3C1 mutations and polymorphisms in a cohort of patients with bilateral adrenal hyperplasia, high arterial blood pressure and/ or biologic hypercortisolism without any stigmata of Cushing syndrome. This multicenter study was set up based on the results of two earlier studies, in which a heterozygous mutant GR was associated with bilateral adrenal hyperplasia both in a patient harboring the hGR $\alpha$ R 469X mutation and in mouse $\mathrm{GR}^{+/-}$model, respectively $(13,14)$. These findings led the authors to hypothesize that the NR3C1 mutations might be an unsuspected cause of bilateral adrenal hyperplasia. For this purpose, 100 patients with incidentally discovered bilateral adrenal hyperplasia, hypertension and/or hypercortisolism without any clinical manifestations of Cushing syndrome were recruited to participate in the study. The inclusion criteria and all clinical, biochemical, endocrine and imaging data were reported (12).

The authors found that 5 in 100 patients harbored novel heterozygous NR3C1 mutations. Two of them, the R469X and the R477S, were located in the DNA-binding domain of hGR $\alpha$, whereas the R491X, the Q501H and the L672P mutations were found in the ligand-binding domain of the receptor. These findings indicate a prevalence of $5 \%$ of NR3C1 mutations in patients with bilateral adrenal hyperplasia (12). Two of the patients harboring the mutant GRs had high blood pressure, whereas four of them met the diagnostic criteria of PGGR (increased urinary free cortisol excretion, normal or high plasma ACTH concentrations and resistance of the HPA axis to the $1 \mathrm{mg}$ dexamethasone suppression test). The authors also showed that patients with defective hGRs had lower potassium levels, lower or normal renin levels and lower aldosterone concentrations, than those without NR3C1 mutations (12). These results may be explained by the elevated secretion of steroids with mineralocorticoid activity, such as cortisol itself, 11-deoxycorticosterone and corticosterone. In in vitro experiments, the investigators exposed fibroblasts obtained from the patients with NR3C1 mutations to dexamethasone for $6 \mathrm{~h}$ and demonstrated that the expression of a glucocorticoid target gene coding for the FKBP5 protein, was lower, suggesting defective glucocorticoid sensitivity (12). Moreover, the authors searched for a possible association between known NR3C1 polymorphisms and bilateral adrenal 
hyperplasia. They only found a high representation of the BCII polymorphism in the 100 participants. Finally, the authors concluded that sequencing of the NR3C1 should be performed in selected patients with bilateral adrenal hyperplasia, endocrinologic findings compatible with PGGR, decreased aldosterone concentrations and decreased potassium levels below $4 \mathrm{mmol} / \mathrm{L}$ (12).

This study provides an estimation of the prevalence of NR3C1 mutations in bilateral adrenal hyperplasia. Although only 26 NR3C1 genetic defects have been identified so far, the percentage of $5 \%$ is indeed a high prevalence, indicating that many cases of NR3C1 neutralizing mutations, deletions or insertions in the general population might be present and missed. Therefore, clinicians should search the NR3C1 for such genetic defects in patients with bilateral adrenal hyperplasia who also have low aldosterone concentrations, serum potassium below $4 \mathrm{mmol} / \mathrm{L}$ and increased urinary free cortisol excretion or resistance of the HPA axis to the $1 \mathrm{mg}$ dexamethasone suppression test. This might be extremely helpful for clinicians to distinguish such cases from preclinical Cushing syndrome or pseudo-Cushing conditions.

The French MUTA-GR study showed that all patients with NR3C1 mutations had low aldosterone concentrations combined with low or normal renin in the presence or absence of hypertension, suggesting an impairment of aldosterone secretion (12). Interestingly, in three of the mutated patients that DOC concentrations were measured, the latter were normal, but this may be due to the known pulsatile and/or marked ultradian variability of steroid hormone secretion. Carriers of R469X had impaired activity of renal 11- $\beta$ hydroxysteroid dehydrogenase type 2 (11- $\beta$ HSD2), probably a result of saturation of the enzyme by the elevated steroids with salt-retaining activity (Fig. 2) (13, 14). Although these results are valid, more studies are needed to justify a role of impaired aldosterone secretion and/or signaling in PGGR.

Another important issue raised in this study was the role of ACTH hyperstimulation on the adrenal glands. In the MUTA-GR study, most of the patients harboring NR3C1 mutations had normal plasma ACTH concentrations, indicating lack of ACTH hyperstimulation on the adrenal cortex, but this could be due to a reset of the HPA axis as that reported in melancholic depression (15) or, again, could be a methodologic issue, granted that the secretion of ACTH is markedly circadian and pulsatile (12). Older studies had demonstrated that ACTH alone may not promote adrenocortical cell growth; yet, simultaneous
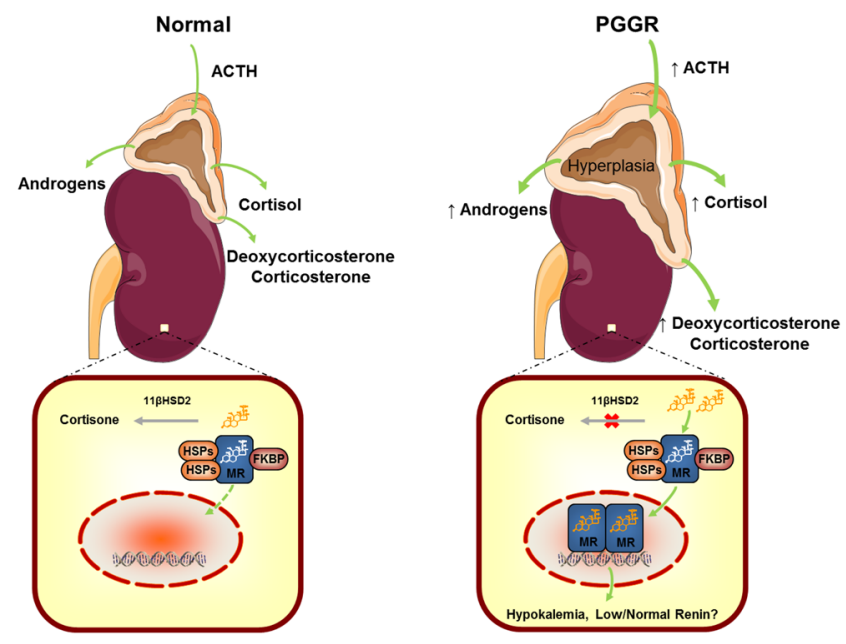

Figure 2

Possible molecular mechanisms leading to activation of mineralocorticoid receptor signaling in the kidney distal convoluted tubule. The presence of markedly elevated levels of salt-retaining corticoids, such as cortisol,

11-deoxycorticosterone and corticosterone, led to the impairment of renal 11- $\beta$ hydroxysteroid dehydrogenase type 2 (11- $\beta$ HSD2) activity and increased activation of the mineralocorticoid receptor in the kidney distal convoluted tubule. 11- $\beta \mathrm{HSD} 2$, 11- $\beta$ hydroxysteroid dehydrogenase type 2; ACTH, adrenocorticotropic hormone; FKBP, immunophilins; HSPs, heat shock proteins; MR, mineralocorticoid receptor; PGGR, primary generalized glucocorticoid resistance.

activation of the adrenal medulla through secretion of NPY or other peptides might synergize in promoting growth of zona fasciculata cortisol-secreting cells (16).

\section{Beyond GR: bilateral adrenal hyperplasia and glucocorticoid resistance in the era of next generation sequencing}

In the French MUTA-GR study, the authors found a significant prevalence of NR3C1 mutations in patients with bilateral adrenal hyperplasia (12). Clinicians should keep in mind that not all cases of PGGR are attributed to NR3C1 mutations, suggesting a possible role of other molecules (GR partners, co-factors, etc.) in the pathogenesis of primary generalized glucocorticoid resistance (11). Novel technologies, such as whole exome/ genome sequencing, could undoubtedly unravel the complex pathways involved in the regulation of tissue sensitivity to glucocorticoids. The 'new' GR interactome is to be extensively investigated. 
Declaration of interest

The authors declare that there is no conflict of interest that could be perceived as prejudicing the impartiality of this commentary.

\section{Funding}

This research did not receive any specific grant from any funding agency in the public, commercial or not-for-profit sector.

\section{References}

1 Nicolaides NC, Kyratzi E, Lamprokostopoulou A, Chrousos GP \& Charmandari E. Stress, the stress system and the role of glucocorticoids. Neuroimmunomodulation 201522 6-19. (https://doi. org/10.1159/000362736)

2 Chrousos GP \& Kino T. Intracellular glucocorticoid signaling: a formerly simple system turns stochastic. Science STKE 2005304 pe48.

3 Nicolaides NC, Galata Z, Kino T, Chrousos GP \& Charmandari E. The human glucocorticoid receptor: molecular basis of biologic function. Steroids 201075 1-12. (https://doi.org/10.1016/j. steroids.2009.09.002)

4 Galon J, Franchimont D, Hiroi N, Frey G, Boettner A, EhrhartBornstein M, O'Shea JJ, Chrousos GP \& Bornstein SR. Gene profiling reveals unknown enhancing and suppressive actions of glucocorticoids on immune cells. FASEB Journal 200216 61-71. (https://doi.org/10.1096/fj.01-0245com)

5 Zannas AS \& Chrousos GP. Epigenetic programming by stress and glucocorticoids along the human lifespan. Molecular Psychiatry 2017 22 640-646. (https://doi.org/10.1038/mp.2017.35)

6 Oakley RH \& Cidlowski JA. The biology of the glucocorticoid receptor: new signaling mechanisms in health and disease. Journal of Allergy and Clinical Immunology 2013132 1033-1044. (https://doi. org/10.1016/j.jaci.2013.09.007)

7 Chrousos G. Q\&A: primary generalized glucocorticoid resistance. BMC Medicine 20119 27. (https://doi.org/10.1186/1741-7015-9-27)

8 Charmandari E \& Kino T. Chrousos syndrome: a seminal report, a phylogenetic enigma and the clinical implications of glucocorticoid signaling changes. European Journal of Clinical Investigation $2010 \mathbf{4 0}$ 932-942. (https://doi.org/10.1111/j.1365-2362.2010.02336.x)

9 Charmandari E. Primary generalized glucocorticoid resistance and hypersensitivity: the end-organ involvement in the stress response. Science Signaling 20125 pt5.

10 Nicolaides NC \& Charmandari E. Chrousos syndrome: from molecular pathogenesis to therapeutic management. European Journal of Clinical Investigation 201545 504-514. (https://doi.org/10.1111/ eci.12426)

11 Nicolaides NC \& Charmandari E. Novel insights into the molecular mechanisms underlying generalized glucocorticoid resistance and hypersensitivity syndromes. Hormones 201716 124-138.

12 Vitellius G, Trabado S, Hoeffel C, Bouligand J, Bennet A, Castinetti F, Decoudier B, Guiochon-Mantel A, Lombes M, Delemer B et al. Significant prevalence of NR3C1 mutations in incidentally discovered bilateral adrenal hyperplasia: results of the French MUTA-GR Study. European Journal of Endocrinology 2018178 411-423. (https://doi.org/10.1530/EJE-17-1071)

13 Bouligand J, Delemer B, Hecart AC, Meduri G, Viengchareun S, Amazit L, Trabado S, Fève B, Guiochon-Mantel A, Young J et al. Familial glucocorticoid receptor haploinsufficiency by nonsense mediated mRNA decay, adrenal hyperplasia and apparent mineralocorticoid excess. PLoS ONE 20105 e13563. (https://doi. org/10.1371/journal.pone.0013563)

14 Michailidou Z, Carter RN, Marshall E, Sutherland HG, Brownstein DG, Owen E, Cockett K, Kelly V, Ramage L, Al-Dujaili ES et al. Glucocorticoid receptor haploinsufficiency causes hypertension and attenuates hypothalamic-pituitary-adrenal axis and blood pressure adaptions to high-fat diet. FASEB Journal 200822 3896-3907. (https://doi.org/10.1096/fj.08-111914)

15 Chrousos GP, Schuermeyer TH, Doppman J, Oldfield EH, Schulte HM, Gold PW \& Loriaux DL. NIH conference. Clinical applications of corticotropin-releasing factor. Annals of Internal Medicine 1985102 344-358. (https://doi.org/10.7326/0003-4819102-3-344)

16 Bornstein SR \& Chrousos GP. Clinical review 104: adrenocorticotropin (ACTH)- and non-ACTH-mediated regulation of the adrenal cortex: neural and immune inputs. Journal of Clinical Endocrinology and Metabolism 199984 1729-1736. (https://doi. org/10.1210/jcem.84.5.5631)

Received 6 June 2018

Revised version received 9 July 2018

Accepted 12 July 2018 